CrossMark

Cite this: Polym. Chem., 2015, 6, 4690

\section{Covalently layer-by-layer assembled homogeneous nanolayers with switchable wettability $\dagger$}

\author{
Fuat Topuz, ${ }^{\star a}$ Martin Möller ${ }^{a}$ and Jürgen Groll ${ }^{b}$
}

Received 6th April 2015, Accepted 15th May 2015 DOI: 10.1039/c5py00515a www.rsc.org/polymers

\begin{abstract}
Layer-by-layer ( $\mathrm{LbL}$ ) assembly is a practical and versatile approach to build up ultrathin hydrogel networks using mostly polyelectrolytes via alternate adsorption of oppositely charged molecules. It has recently been applied covalently by means of many different types of molecules, particularly those having low molecular weights or linear polymer structures. Using isocyanate (NCO) end-functional star-type polyethers (NCO-sP(EO-stat-PO)) in such covalent assemblies is a great challenge, since they are prone to form a smooth but non-reactive layer that precludes chemisorption of a subsequent layer. To overcome this problem, we developed a protocol where oligomers (e.g., dimers, trimers) act as building blocks for a monolayer instead of single star shaped molecules. Since these are larger and multifunctional but still flexible, smooth layers thicker than a monomolecular film (ca. $10 \mathrm{~nm}$ ) result with sufficient mobility of the building blocks to bear enough reactive groups for covalent binding of a subsequent layer. As a second component for the chemical LbL layer buildup, a high molecular weight copolymer of vinylformamide/ vinylamine (PVFA-CO-PVAm) was used. The first layer was obtained by treating aminosilylated surfaces with NCO-s(EO-stat-PO) followed by incubation with PVFA-CO-PVAm and chemical cross-linking with the first layer via urea links. The cycle was repeated to achieve the desired layer growth, and the resulting layers were characterized by ellipsometry, contact angle analysis, X-ray photoelectron spectroscopy (XPS), and scanning force microscopy (SFM). The amorphous structures of the polymers were revealed by WAXS analysis, suggesting the lack of the long-range order, which led to structural degree of freedom available to the polymer (i.e., molecular flexibility) on the surface. Thus, multilayers were obtained with homogeneous structure together with low roughness values, and the water contact angles of the layers switched between 37 and $45^{\circ}$ depending on the terminal layer. The layers were stable over three months under humid conditions during which no significant changes could be observed in thickness and hydrophilicity.
\end{abstract}

\section{Introduction}

The precise control of surface coatings is intriguing for the design of functional surfaces and interfaces due to its importance for surface properties that could be particularly tailored for a specific application. ${ }^{1-3}$ In that context, bottom-up approaches provide such a unique control on the surface composition and properties by adjusting the outermost layer that can switch off/on the reactivity or hydrophilicity of the coatings

${ }^{a}$ DWI-Leibniz Institute for Interactive Materials, RWTH Aachen University, Forkenbeckstrasse 50, 52074 Aachen, Germany.E-mail: fuat.topuz@rwth-aachen.de ${ }^{b}$ Department of Functional Materials in Medicine and Dentistry, University of Würzburg, Pleicherwall 2, 97070 Würzburg, Germany

$\dagger$ Electronic supplementary information (ESI) available: The results related to the optimization of pre-coating parameters are available. See DOI: 10.1039/ c5py00515a towards proteins or cells. One of the most common ways of growing the layers through the bottom-up technique is layerby-layer (LbL) assembly, which uses different building blocks, such as polyelectrolytes, nanoparticles, and biomacromolecules, and various techniques (e.g., coordination, biologically specific interactions, electrostatic, hydrophobic, hydrogen bonds, and host-guest interactions). ${ }^{4-15}$ Electrostatic LbL deposition is the most used approach to grow layers to the desired thickness using polyionic complexes on different kinds of materials (e.g., nanogels, hydrogels, surface coating). ${ }^{16-21}$ However, these assemblies are susceptible to environmental conditions and can disintegrate with $\mathrm{pH}$ changes, disrupting ionic interactions between the layers. ${ }^{4}$ Apart from electrostatic interactions and as well as other noncovalent methods, it is possible to grow layers up to thin hydrogel films using functional molecules through the covalent LbL technique. In this case, multilayer formation 
could be achieved through chemical bonds between reactive molecules, and the method yields mechanically strong and chemically stable layers even under harsh conditions at which electrostatically assembled multilayers cannot endure. In that context, many attempts have already been made using different building blocks. Kohli et al. (2000) reported multilayer formation through LbL and the spontaneous growth approach using isocyanate (-NCO) and amine-terminated low molecular weight compounds. ${ }^{22}$ Due to the reactivity of NCO groups towards amino $\left(\mathrm{NH}_{2}\right)$ groups along with a rigid molecular structure of the used molecules, multilayer formation was easily accomplished, and by repeating the cycle many times, the coating thickness was manually tailored to the desired values. Contrary to the previous example, Seo et al. (2010) reported multilayer films using the functional linear polymers, poly(pentafluorophenyl 4-vinylbenzonate) and poly(allylamine), through amide bonds. ${ }^{23}$ Those approaches and many other studies reported covalently bound LbL assemblies, which generally rely on the strategy that mainly uses small molecules, mostly hydrophobic, or linear polymers, and some of them may not be compatible for biological use in terms of hydrophobicity of the layers, which result in uncontrolled adsorption of proteins and protein mediated adhesion of cells. ${ }^{23-28}$ Furthermore, high roughness values of such coatings in contrast to electrostatic assemblies are a critical problem, since some of them are far away from uniform morphology.

Star-type polymers with multiple arms connected to the central core are of interest for surface coating applications, since they can provide a high polymer segment density on the surface ensuring high surface coverage. Apart from the hydrophilicity of the polymers used, this is one critical criterion for preventing uncontrolled adsorption of proteins or also protein-mediated adhesion of cells. ${ }^{29}$ Furthermore, terminal groups of such molecules can be functionalized with reactive moieties for selective interaction of ligands that are automatically endowed with a molecular spacer. It would be of general interest to investigate the structural and morphological influences of LbL assemblies based on those branched molecules and linear polymers and gain insights into their formation and properties. However, the layer growth from such molecules is challenging as it crucially depends on the ability to control end-group orientation. For instance, we have previously reported that amphiphilic isocyanate-terminated six armed star shaped prepolymers with $80 \%$ ethylene oxide and $20 \%$ propylene oxide in the backbone (NCO-sP(EO-stat-PO)) with a molecular weight of 12 or $18 \mathrm{kDa}$ can be used for thin hydrogel layer preparation from aqueous solutions with straightforward control over layer thickness through 3D crosslinking in the polymer layer. However, in the case of adsorption of these molecules on amino-functional surfaces from non-aqueous solution under an inert gas atmosphere, the functional groups react rapidly and the molecules form a flat monolayer with a maximum thickness of $\sim 1.2 \mathrm{~nm}$, which corresponds to $\sim 10 \%$ of the size of the molecule. ${ }^{30}$ When such a surface is incubated in an $\mathrm{SP}(\mathrm{EO}-$-stat-PO) solution again, no significant thickness change could be detected due to chemisorption of the reactive distal ends of the star molecules, resulting in a hydrophilic but non-functional layer. The high flexibility of the $\mathrm{sP}(\mathrm{EO}-$ stat-PO) polymers creates little steric hindrance so that all isocyanate groups may react with aminogroups on the surface. In the presence of water, hydrolysis of isocyanates and subsequent covalent coupling of star molecules to larger aggregates creates sufficient steric hindrance to allow three-dimensional cross-linking of the coating.

Hence, in the present study, we changed the concept of the layer growth with the aim of controlling the density of functional groups in each layer to allow for LbL assembly. Firstly, we use a combination of $12 \mathrm{kDa}$ star-shaped NCO-sP (EO-stat-PO) polymers and high molecular weight (340 kDa) linear copolymers of vinylformamide/vinylamine (PVFA-coPVAm) (Fig. 1) in order to have a better control of the functional group density on the surface. Secondly, we incubated the substrates with NCO-sP(EO-stat-PO) solutions in a clean room under ambient conditions in the presence of humidity to allow the formation of aggregates, e.g., dimers and trimers on the substrate. We hypothesized that this would generate sufficient steric hindrance to prevent all NCO groups from reacting with the surface and thus result in a thick layer that presents functional groups on the surface for subsequent layer chemisorption while leaving enough mobility for the aggregates to form smooth and homogeneous layers. Finally, the high molecular weight of the PVFA-co-PVAm was chosen to ensure that chemisorption would occur and, in addition, a sufficient amount of amine groups would remain for the subsequent layer. The resulting layers were characterized by ellipsometry, contact angle analysis, X-ray photoelectron spectroscopy (XPS), and scanning force microscopy (SFM).

\section{Experimental part}

\section{Materials}

Glass substrates $(\varnothing \sim 15 \mathrm{~mm})$ and silicon (100) wafers were purchased from VWR International GmbH (Germany) and CrysTec GmbH (Germany), respectively. THF (Merck) was dried over $\mathrm{LiAlH}_{4}$, distilled under argon, and transferred into a glove box. 3-Aminopropyl-trimethoxysilane was obtained from Sigma Aldrich and used as received. Six-armed star-shaped $\mathrm{OH}$ terminated prepolymers with a backbone of statistically copolymerized $80 \%$ ethylene oxide and $20 \%$ propylene oxide and a molecular weight of $12000 \mathrm{~g} \mathrm{~mol}^{-1}(\mathrm{PDI}=1.15)$ were obtained from Dow Chemical Co. (The Netherlands) and hydroxyl endgroups were transformed into isocyanate (NCO) groups. A copolymer of vinylformamide/vinylamine (PVFA-co-PVAm, Lupamin 9050$)$ of high molecular mass $\left(M_{\mathrm{w}}=340 \mathrm{kDa}\right)$ was purchased from BASF Chemicals. The solid content of PVFA$c o$-PVAm was reported to be in the range of $16-19 \%$ in water and $\mathrm{pH}$ of 7-9. Sessile water was used in all experiments, unless otherwise stated. 

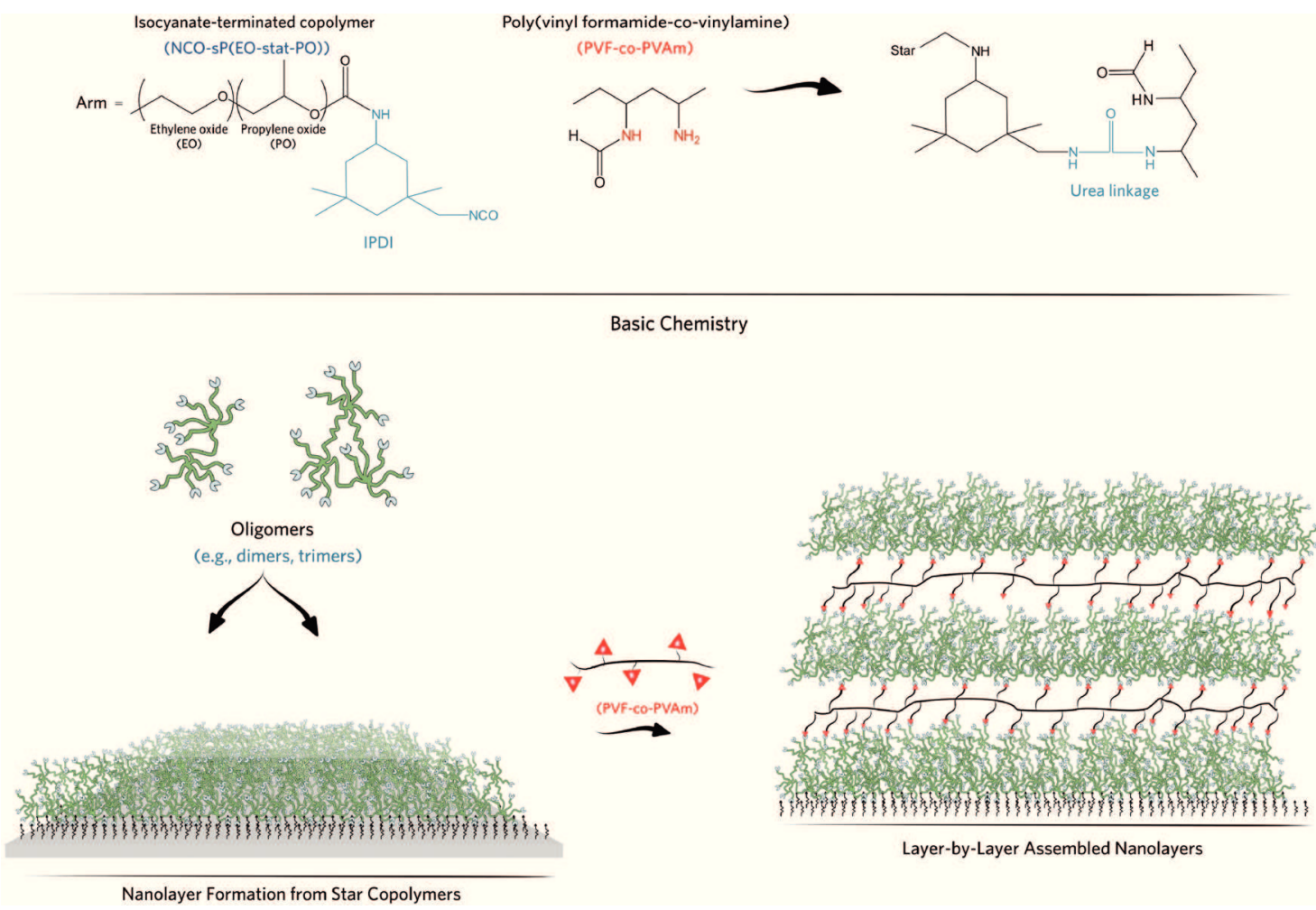

Fig. 1 Prepolymers used in the fabrication of LbL growth nanolayers and the cartoon illustration of multilayer formation.

\section{Synthesis of NCO-sP(EO-stat-PO)}

The preparation of the star polymer has been described elsewhere. ${ }^{31}$ Briefly, $\mathrm{OH}$ terminated star polyols $\left(M_{\mathrm{n}}=12000 \mathrm{~g}\right.$ $\mathrm{mol}^{-1} ;$ PDI $\left.=1.15\right)$ were functionalized through reaction with 12 times excess isophorone diisocyanate (IPDI) in a solvent free process at $50{ }^{\circ} \mathrm{C}$ for four days under an inert atmosphere. The excess of IPDI was removed by a short path distillation.

\section{Gas-phase aminosilylation}

The substrates were cleaned using ultra-sonication with acetone, isopropanol, and de-ionized water for $5 \mathrm{~min}$ and dried by nitrogen blowing. Activation of the surfaces was achieved by treatment with UV/ozone for $30 \mathrm{~min}$ at $5 \mathrm{mbar}$. After that step, water-contact angles of the substrates were below the detection limit. The substrates were immediately used for amino-functionalization.

After the activation process with ozone, the substrates were held in a desiccator with $100 \mu \mathrm{L}$ 3-aminopropyl-trimethoxysilane at 5 mbar for $1 \mathrm{~h}$. After removing the 3-aminopropyl-trimethoxysilane, the substrates were kept under high vacuum $\left(\sim 10^{-2}\right.$ mbar $)$ for $1 \mathrm{~h}$ to remove unbound silane molecules.

\section{Surface coating}

The precise amount of NCO-terminated $\mathrm{SP}($ EO-stat-PO $)\left(M_{\mathrm{n}}=\right.$ $12000 \mathrm{~g} \mathrm{~mol}^{-1}$; PDI = 1.15) was dissolved under an inert gas atmosphere in dry THF, and then the solution was transferred to the clean room and dropped onto aminosilylated surfaces.
The first layer was obtained by $30 \mathrm{~min}$ incubation (dip coating) under ambient conditions and then unreacted prepolymers were removed by washing with dry THF. Afterwards, aqueous solution of a copolymer PVFA-co-PVAm was dropped onto the first layer, and the slides were incubated for $30 \mathrm{~min}$. Following that step, the slides were washed with water. This cycle was repeated to achieve the desired thickness.

\section{Scanning force microscopy (SFM)}

The morphology of the layers was investigated by a tapping model SFM (NanoScope V, Digital Instruments Veeco Instruments, Santa Barbara, CA) under ambient conditions. Commercially available standard silicon cantilevers (PPP-SEIH-W from Nanosensors) with a spring constant of 5-37 $\mathrm{N} \mathrm{m}^{-1}$ and an oscillation frequency of $\sim 125 \mathrm{kHz}$ were used. The data were processed using Gwyddion software Analysis, v.2.38.

\section{X-Ray photoelectron spectroscopy (XPS)}

XPS measurements were carried out in an Ultra Axis ${ }^{\mathrm{TM}}$ spectrometer (Kratos Analytical, Manchester, UK). The samples were irradiated with monoenergetic $\mathrm{Al} \mathrm{K} \mathrm{K}^{*} 1,2$ radiation $(1486.6 \mathrm{eV})$, and the spectra were recorded at a power of $144 \mathrm{~W}(12 \mathrm{kV} \times$ $12 \mathrm{~mA})$. The aliphatic carbon $(\mathrm{C}-\mathrm{C}, \mathrm{C}-\mathrm{H})$ at a binding energy of $285 \mathrm{eV}$ (C 1s photoline) was used to determine the charging. The spectral resolution, i.e., the full width at half-maximum (fwhm) of the ester carbon from PET, was better than $0.68 \mathrm{eV}$ for the elemental spectra. The elemental concentration is given in atom\%. 


\section{Ellipsometry}

Layer thickness was measured using a MM-SPEL-VIS ellipsometer from OMT. Polymer-coated silicon wafers were examined with a spectral method in the wavelength range of 450-900 nm. The azimuthal angle $(\varphi)$, which corresponds to the angle between the plane of incidence and one of the axes of symmetry of the sample, was kept at $15^{\circ}$. The integration time was dependent on the layer thickness and the resulting signal intensity. The main reason for systematic errors during the measurements is the correct position of the sample, which may lead to uncertainties in both the angle of incidence and the azimuthal angle. However, this is minimized by sequential measurements using the same settings. Furthermore, each sample was measured over three different areas, which averaged over the area of $3 \times 5 \mathrm{~mm}$. The thicknesses of the layers were obtained as relative values to the amino-functional substrate.

\section{Wide-angle X-ray scattering (WAXS)}

WAXS was done using an Empyrean setup from PANalytical. A $\mathrm{Cu}$ X-ray tube (line source of $12 \times 0.04 \mathrm{~mm}^{2}$ ) provided $\mathrm{Cu} \mathrm{K} \alpha$ radiation with $\lambda=0.1542 \mathrm{~nm}$. The $\mathrm{K} \beta$ line was removed by a $\mathrm{Ni}$ filter. The source and the detector moved in the vertical direction around a fixed horizontal sample. After passing a divergence slit of $1 / 8^{\circ}$ and an anti-scatter slit of $1 / 4^{\circ}$, the beam reached the sample at the center of a phi-chi-z stage. In the Bragg-Brentano geometry used, the beam was refocused at a secondary divergence slit of $1 / 4^{\circ}$. Finally, the signal was recorded using a pixel detector $(256 \times 256$ pixels of $55 \mu \mathrm{m})$ as a function of the scattering angle $2 \theta$. Subsequently, the peak positions were calculated from $q=2 \pi / d=(4 \pi / \lambda) \sin \theta$, in which $q$ is the scattering vector. The detector was used in a scanning geometry that allowed all rows to be used simultaneously.

To reduce the background, the divergent beam perpendicular to the scattering plane was controlled by a mask of $4 \mathrm{~mm}$ restricting the width of the beam at the sample position to about $10 \mathrm{~mm}$. In addition, the perpendicular divergence was restricted by Soller slits to angles $\leq 2.3^{\circ}$. For each new measurement the height of the (powder) sample was optimized. Scans were performed with $2 \theta$, the detector axis, moving at twice the rate of the $\theta$-axis of the incident beam. The calibration was checked using a Si reference sample.

\section{Contact angle analysis}

Contact angle analyses were performed by sessile drop measurements with a goniometer G40 (Krüss, Hamburg, Germany). For each sample, three droplets were measured, and the average data are presented with a standard deviation.

\section{Results and discussion}

Isocyanate (NCO) groups are highly reactive groups with a unique in situ network forming chemistry. ${ }^{32}$ In aqueous solutions, they can instantly hydrolyse into amine over unstable carbamic acid and later can react with free NCO groups that ultimately yield the hydrogel network. With a combination of this unique chemistry with branched star architecture; we previously developed a functional ultrathin hydrogel layer coating with tunable thickness, but not very precisely, with parameters employed in coating preparation (e.g., spin-coating speed and polymer concentration). ${ }^{30}$ This platform showed promising results as protein resistance surfaces and in the prevention of uncontrolled cell adhesion. Furthermore, this sole platform could be simultaneously functionalized with peptides or proteins for the intended biological use. ${ }^{29}$ Surface wettability of polymers is a prominent parameter for the assembly of the molecules on the surface to attain uniform coating and it is affected by various factors, including surface-polymer interactions, solvent-polymer interactions, molecular flexibility (e.g., crystallinity, persistence length) and shape. Rigid polymers (e.g., deoxyribonucleic acid (DNA) and polyisocyanides) are not desirable for the fabrication of LbL assemblies due to their high persistence length $(\xi)$, a measure of lengthwise thermal bending fluctuations, yielding partly covered layers. Similarly, crystalline or semi-crystalline polymers are also not preferable for LbL systems due to their degree of structural order, which yields particular alignment of the crystalline zones, ultimately yielding patterned layers. To determine the configuration of the polymers, whether they have crystalline phases (polymorphism) or not, wide-angle X-ray scattering analyses were performed at bulk and in solvents. X-ray diffraction data on the bulk polymers are shown in Fig. 2(a). PVFA-coPVAm displays small crystalline peaks at $2 \theta$ of $20.6^{\circ}, 26.5^{\circ}$, $32.3^{\circ}, 39.5^{\circ}$, and $46.1^{\circ}$, together with a broad amorphous peak at $23.8^{\circ}(d$-spacing $\sim 3.73 \AA)$, while the $\mathrm{sP}(\mathrm{EO}-$ stat-PO) is a highly amorphous polymer having a broad peak centred at $20^{\circ}$ ( $d$-spacing $\sim 4.4 \AA$ A). Likewise, both polymers displayed highly broad peaks in respective solvents, suggesting their highly amorphous configurations during the coating process (Fig. 2(b)). Thus, the lack of the long-range order leads to structural degree of freedom available to the used polymers.

Prior to coatings, the substrates (glass substrates and silicon wafers) were activated by UV-ozone treatment for $30 \mathrm{~min}$. After that step, the contact angle was lower than the detection limit of the method $\left(<10^{\circ}\right)$ (see ESI, $\uparrow$ Fig. S1). Activated surfaces were aminosilylated with 3-aminopropyltrimethoxysilane via the gas phase in an exicator at 5 mbar for
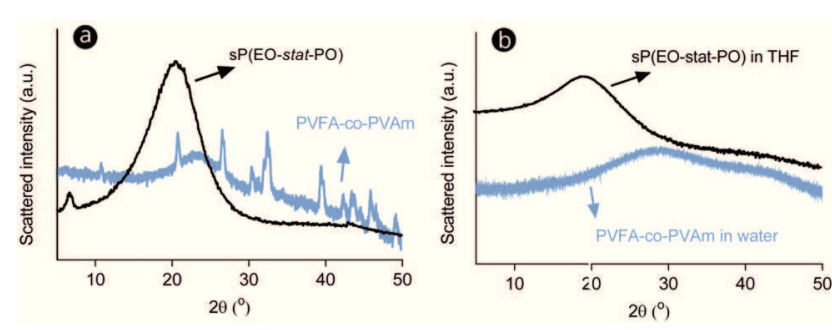

Fig. 2 (a) WAXS patterns of bulk polymers: lyophilized PVFA-co-PVAm and $\mathrm{SP}(\mathrm{EO}-$-stat-PO) and (b) in the solvents: $\mathrm{SP}(\mathrm{EO}-$-stat-PO) in THF and PVFA-CO-PVAm in water. 
$1 \mathrm{~h}$, and then $1 \mathrm{~h}$ under high vacuum to remove unbound silane molecules. Silane treatment of the surfaces resulted in an amino-terminated surface (Fig. S2 $\dagger$ ). The formation of the first layer was achieved by dip coating of the aminosilyated surfaces with $10 \mathrm{mg} \mathrm{mL}^{-1} \mathrm{sP}($ EO-stat-PO) in anhydrous THF. Treated surfaces were incubated at RT for $30 \mathrm{~min}$, and unreacted prepolymers were removed by washing with anhydrous THF. The first layer formation was evidenced by ellipsometry and contact angle measurements, revealing a contact angle of $45^{\circ}$ and a thickness of about $12 \mathrm{~nm}$, while the corresponding values for amino-functional surfaces were found to be $65^{\circ}$ and $\sim 1 \mathrm{~nm}$. The layer thickness of the amino-functional surface was supported by the theoretical nominal thickness of the silane molecule, calculated using ChemBio3D software, and suggests precise monolayer formation (Fig. S2, ESI $\dagger$ ). The thickness obtained from the first layer is $10 \mathrm{~nm}$ and thus almost 10 times larger than the reported value for NCO-sP(EOstat-PO) molecules chemisorbed onto aminosilanized glass substrates under an inert gas atmosphere $(1.2 \mathrm{~nm})$, thus indicating that by incubation for 30 minutes under ambient conditions, aggregates (e.g., dimers, trimers) adsorb onto and/or form on the surface. As this layer thickness does relate to a stretched radius of a NCO-SP(EO-stat-PO) molecule, we conclude that no larger aggregates are present but trimers and tetramers which are sufficiently flexible during adsorption. That also results in enough mobility to form a thick and homogeneous layer to allow subsequent layer through chemical bonds as illustrated in Fig. 1. The second layer was prepared by treating the reactive first layer with PVFA-co-PVAm (solid content $=16-19 \mathrm{wt} \%$ in water) for $30 \mathrm{~min}$, and unbound chemicals were removed by washing with an excess of water. Following that step, thickness and contact angle analyses revealed considerable changes; contact angle was decreased from $45^{\circ}$ to $37^{\circ}$, and the thickness was increased $\sim 2 \mathrm{~nm}$. This cycle was repeated to obtain multilayers in a stepwise manner. Fig. 3 shows the hydrophilicity changes by water

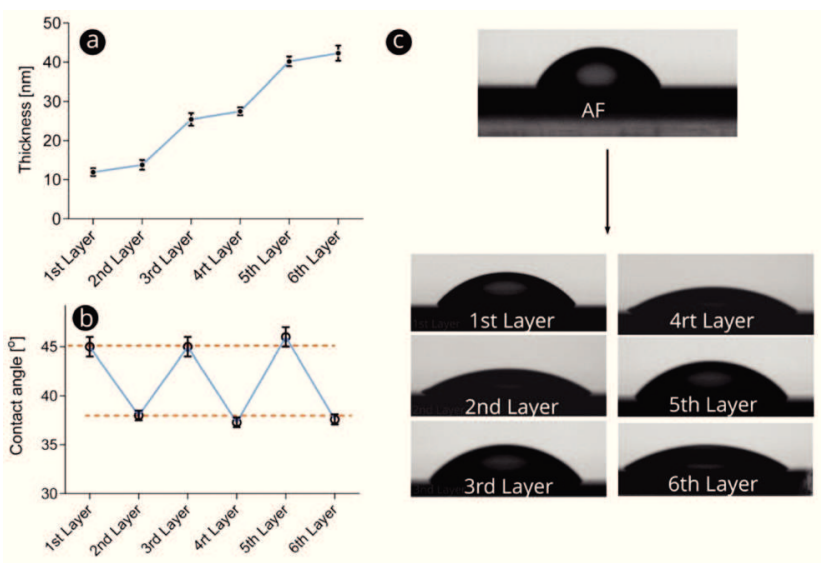

Fig. 3 Influence of layer growth via LbL on coating thickness and contact angle (a, b). Panel (c) respectively shows wettability on the aminosilyated surface and the coated surfaces. droplet analyses. The amino-silylated surface is rather hydrophobic with a contact angle of $\sim 65^{\circ}$ driven by the alkyl chain of the silane compound. After treating the aminosilylated surface with NCO-sP(EO-stat-PO) prepolymers, the contact angle significantly dropped by $\sim 20^{\circ}$. Fig. 3(c) shows that the water droplet spreads on the $1^{\text {st }}$ layer surface. With further incorporation of the copolymer of vinylformamide/vinylamine (PVFA-co-PVAm) as a second layer, spreading of the droplet increased and the contact angle decreased by $\sim 7^{\circ}$. Star molecules are a copolymer of hydrophilic ethylene oxide (EO) and hydrophobic propylene oxide (PO), and the IPDI-derived terminal groups have also hydrophobic character. That explains contact angle differences between SP(EO-stat-PO) and (PVFAco-PVAm) terminal layers. Yet, numerous studies of our research group support that $\mathrm{SP}(\mathrm{EO}-$ stat-PO)-based thin hydrogel layers are efficient in minimizing protein adsorption even at this contact angle. ${ }^{29}$ With again treating the coating with star polymers, layer growth was successfully accomplished.

We use X-ray photoelectron spectroscopy to quantitate the elemental composition of the layers. Fig. 4 shows the XPS spectra of the layers. Although both prepolymers have an excess of carbon (C) and oxygen (O) atoms, they have significantly different nitrogen $(\mathrm{N})$ atom contents. As seen in Fig. 4, where the nitrogen atom that appeared at $\sim 400 \mathrm{eV}$ increases with PVFA-co-PVAm incorporation and the peak of Si disappears with increased layer thickness, suggesting the formation of multilayer films since the XPS at the used angle can scan over $10 \mathrm{~nm}$ depth. The atomic compositions of the respective layer shown in Table 1 show higher $\mathrm{C}$ atom and lower Si contents with the increase of layer number. The thick and functional layer formation from NCO-sP(EO-stat-PO) in dry THF could only be elucidated with the pseudo-monomolecular layer of the aggregates. That is, adsorption of aggregates results in a thick and fully covered layer followed by the chemical reactions between $\mathrm{NCO}$ and the terminal $\mathrm{NH}_{2}$ on the surface. A similar phenomenon is also responsible for 3D hydrogel film formation of NCO-sP(EO-stat-PO) in aqueous solutions; once the NCO-sP(EO-stat-PO)s contact with water, hydrolysis of $\mathrm{NCO}$ groups into $\mathrm{NH}_{2}$ groups over unstable carbamic acid and instant reaction of $\mathrm{NCO}$ with formed $\mathrm{NH}_{2}$ groups lead to oligomer formation. When those oligomers adsorb onto amino-functional surfaces they covalently bind to the surfaces through urea links and subsequent aggregates react with the formed layer that ultimately yields $3 \mathrm{D}$ hydrogel layers.

Scanning force microscopy (SFM) was used to explore the surface morphology of coatings as topological and phase profiles for the characterization of film growth (Fig. 5). After the coating of amino-functional surfaces with NCO-sP(EO-stat-PO) prepolymers, SFM topology images suggest the formation of a highly uniform layer (roughness $\left(S_{\mathrm{q}}\right)=3 \AA( \pm 0.25)$ ). In addition to topological analysis of the layers on surface roughness, SFM phase images provide compositional imaging of the coatings regardless of its roughness; i.e., homogeneous coatings give a single contrast while heterogonous coatings yield contrast gradients. The SFM phase image of the first layer shows a single contrast, suggesting the homogeneous layer formation. 

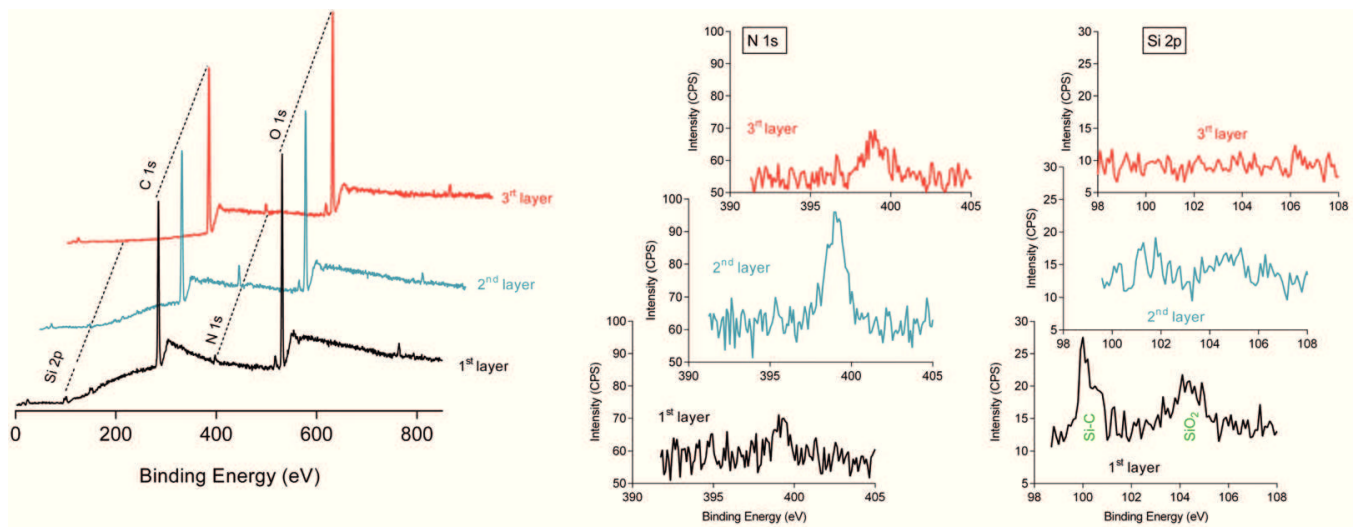

Fig. 4 XPS spectra of the layers up to 3 layers, and XPS N 1s and Si 2p narrow scans of the respective layers.

Table 1 XPS analysis of coated layers, showing that carbon as atomic percentage increases while silicon content decreases with increasing layer number

\begin{tabular}{lllll}
\hline & C 1s & N 1s & O 1s & Si 2p \\
\hline $1^{\text {st }}$ layer & 66.47 & 2.94 & 25.83 & 4.76 \\
$2^{\text {nd }}$ layer & 67.75 & 4.74 & 26.05 & 2 \\
$3^{\text {rd }}$ layer & 70.45 & 2.85 & 26.67 & 0
\end{tabular}

The values represent atomic compositions over $100 \%$.

However, after the second layer formation, $S_{\mathrm{q}}$ is increased to $15 \AA( \pm 2)$ due to clusters of aggregates seen in the topological profile. That is also seen in the phase image showing patterned clustering of the linear polyamine compound with a contrast gradient on the $1^{\text {st }}$ layer. Most interestingly, after subsequent $\mathrm{sP}(\mathrm{EO}-$ stat-PO) coating, no remarkable change in
$S_{\mathrm{q}}$ was observed. The phase image of the $3^{\text {rd }}$ layer shows a decrease of the contrast gradient after the coating with oligomers. Those values present the formation of a uniform coating with low $S_{\mathrm{q}}$ and low surface homogeneity index (SHI), which represents the ratio of roughness to surface thickness. SHI for electrostatics based LbL assemblies is very low and varies in the range of $1-5 \%$, but for covalently grown layer assemblies, it is higher, even reaching $50 \%{ }^{23}$ due to non-uniform coating. This is probably due to the fact that the preparation of layers involves short reaction times (in $10 \mathrm{~min}$ for each layer). Although this short time seems to be an advantageous property for the fabrication of such assemblies within a short time, it inevitably results in the formation of partly covered layers. In this study, we prolong the coating time to $30 \mathrm{~min}$ to avoid such effects and to allow for reorganization of the NCO-sP(EOstat-PO) aggregates to smoothen defects occurring in the second layer prepared from a linear polymer. For our coating system, SHI (surface homogeneity index; roughness/layer thick-

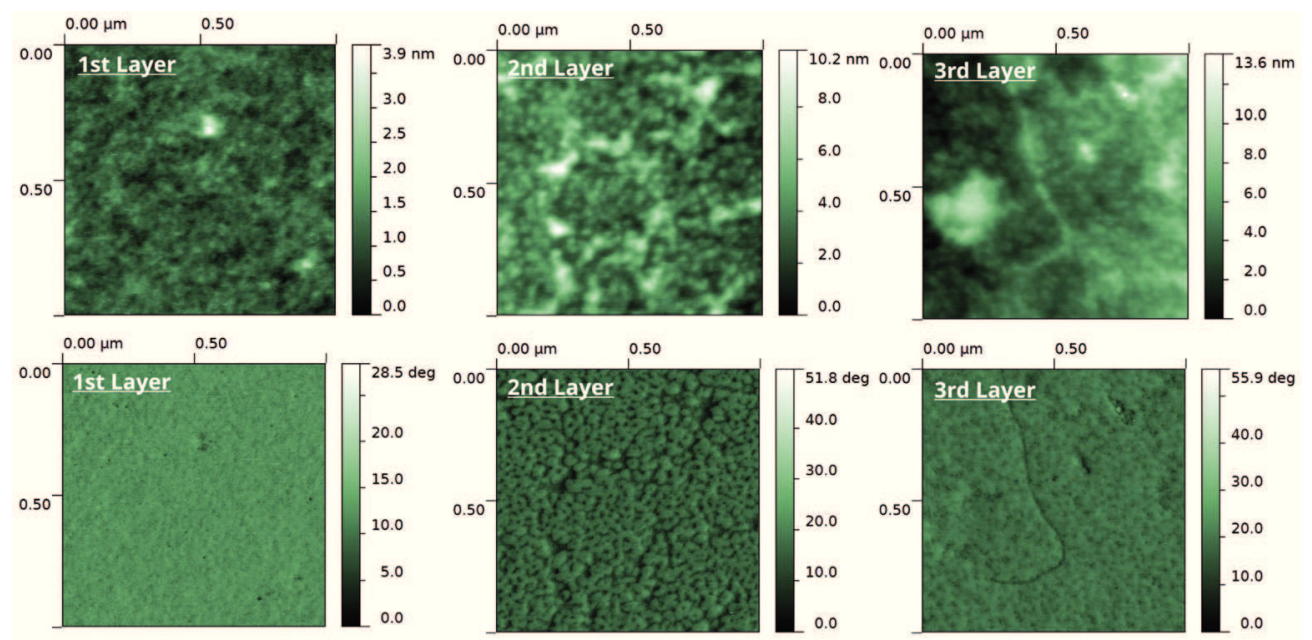

Fig. 5 SFM images of sequentially assembled layers on silicon wafers. Topological (upper row) and phase (lower row) SFM images of the layers: $1^{\text {st }}, 2^{\text {nd }}$ and $3^{\text {rd }}$ layers respectively correspond to the $\mathrm{SP}(\mathrm{EO}-$-stat-PO) nanolayer assembled on 3-aminopropyl)trimethoxysilane coated silicon wafers, sP(EO-stat-PO)-(PVFA-co-PVAm) double-layer and sP(EO-stat-PO)-(PVFA-co-PVAm)-sP(EO-stat-PO) triple layer. 


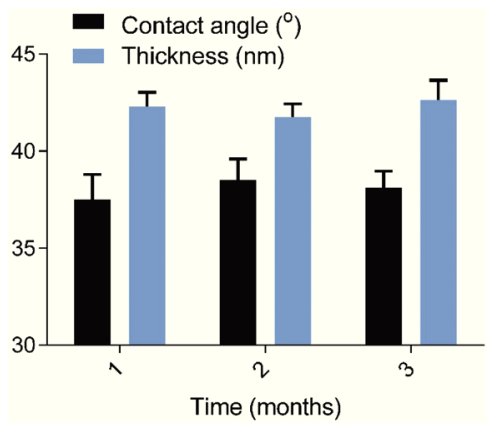

Fig. 6 Stability of the coated surfaces (6 layers) in terms of thickness $(\mathrm{nm})$ and contact angle changes during 3 months storage under humid conditions at $37^{\circ} \mathrm{C}$.

ness) was found to be less than $5 \%$, and decreased with increasing the layer number, suggesting the formation of highly uniform coating.

Using multilayer films for biological applications requires the biocompatibility and chemical stability of the coating over a particular time. Previous studies on sP(EO-stat-PO) layers suggest their biocompatibility for many cell types and even hemocompatibility against plasma proteins and blood cells. ${ }^{33}$ Their stability in aqueous solution over cell experiments for short times (until two weeks) has previously been reported. ${ }^{34}$ Thus we herein have investigated the stability of the layers under humid conditions and $37^{\circ} \mathrm{C}$ over three months, which is good to evaluate their stability over storage for longer times (Fig. 6). The layers kept under humid conditions were taken out and washed with water to remove dust and disintegrated molecules and dried under a stream of nitrogen atmosphere. The multilayers were analysed with ellipsometry and goniometry to obtain the thickness, and the contact angle of the LbL assembly over each month with a standard deviation of 5 days revealed that no significant changes occurred in the surface composition in terms of contact angle and thickness under humid conditions, suggesting the chemical stability of the coatings.

\section{Conclusions}

We successfully demonstrated layer-by-layer growth through alternating chemisorption of isocyanate functional low molecular weight (12 kDa) star shaped polyethers (NCO-sP(EO-stat$\mathrm{PO})$ ) and a high molecular weight $(340 \mathrm{kDa})$ aminofunctional linear polymer ((PVFA-co-PVAm)) via the dip coating technique. Several methods were used to elucidate the property-structure relationship of the resulting layers in terms of composition, morphology, thickness and hydrophilicity. The thickness of the resulting film was easily modulated by the applied cycle number. In contrast to linear polymer assemblies reported in the literature, which show a general tendency for an increase of roughness with the layer number due to the cluster formation, sP(EO-stat-PO) oligomer formation on the surface not only allows the possibility of chemical LbL buildup but also supports the formation of smooth and uniform coating and prevents the rise in surface roughness. The terminal layer defined the general properties of the layer and may easily be modulated via further bio-activation before in situ hydrolysis of NCO groups of star molecules into the nonreactive amine groups in aqueous solutions. The LbL assemblies were stable for three months under humid conditions and showed no significant changes in thickness and hydrophilicity, suggesting its stability during long time storage. All these advantages make this approach a promising system to obtain covalently LbL assembled nanolayers together with homogeneous structures and switchable wettability for possible biomedical applications.

\section{Acknowledgements}

F.T. thanks the Marie Curie ITN project Hierarchy (contract: PITN-2007-215851) for financial support.

\section{Notes and references}

1 Y. Li, X. Wang and J. Sun, Chem. Soc. Rev., 2012, 41, 59986009.

2 P. T. Hammond, Adv. Mater., 2004, 16, 1271-1293.

3 G. K. Such, A. P. R. Johnston and F. Caruso, Chem. Soc. Rev., 2011, 40, 19-29.

4 T. A. Taton, R. C. Mucic, C. A. Mirkin and R. L. Letsinger, J. Am. Chem. Soc., 2000, 122, 6305-6306.

5 Y. M. Lvov, Z. Q. Lu, J. B. Schenkman, X. L. Zu and J. F. Rusling, J. Am. Chem. Soc., 1998, 120, 4073-4080.

6 Y. Lvov, K. Ariga, I. Ichinose and T. Kunitake, J. Am. Chem. Soc., 1995, 117, 6117-6123.

7 J. Schmitt, G. Decher, W. J. Dressick, S. L. Brandow, R. E. Geer, R. Shashidhar and J. M. Calvert, Adv. Mater., 1997, 9, 61-65.

8 Z. Y. Zhang, S. Maji, A. B. D. Antunes, R. De Rycke, Q. L. Zhang, R. Hoogenboom and B. G. De Geest, Chem. Mater., 2013, 25, 4297-4303.

9 G. Decher, J. D. Hong and J. Schmitt, Thin Solid Films, 1992, 210, 831-835.

10 Y. Lvov, G. Decher and H. Moehwald, Langmuir, 1993, 9, 481-486.

11 N. A. Kotov, Nanostruct. Mater., 1999, 12, 789-796.

12 W. B. Stockton and M. F. Rubner, Macromolecules, 1997, 30, 2717-2725.

13 Y. Zhang and W. Cao, New J. Chem., 2001, 25, 483486.

14 W. Muller, H. Ringsdorf, E. Rump, G. Wildburg, X. Zhang, L. Angermaier, W. Knoll, M. Liley and J. Spinke, Science, 1993, 262, 1706-1708.

15 X. Wang, Z. Jiang, J. Shi, Y. Liang, C. Zhang and H. Wu, ACS Appl. Mater. Interfaces, 2012, 4, 3476-3483. 
16 G. Decher, Science, 1997, 277, 1232-1237.

17 Y. W. Tang, Y. Zhao, H. X. Wang, Y. Gao, X. Liu, X. G. Wang and T. Lin, J. Biomed. Mater. Res., Part A, 2012, 100A(8), 2071-2078.

18 N. Akbay, J. R. Lakowicz and K. Ray, J. Phys. Chem. C, 2012, 116, 10766-10773.

19 X. Zhang, H. Chen and H. Zhang, Chem. Commun., 2007, 1395-1405.

20 J. B. Schlenoff, Langmuir, 2009, 25, 14007-14010.

21 N. Joseph, P. Ahmadiannamini, R. Hoogenboom and I. F. J. Vankelecom, Polym. Chem., 2014, 5, 1817-1831.

22 P. Kohli and G. J. Blanchard, Langmuir, 2000, 16, 46554661.

23 J. Seo, P. Schattling, T. Lang, F. Jochum, K. Nilles, P. Theato and K. Char, Langmuir, 2010, 26, 1830-1836.

24 S. L. Bechler and D. M. Lynn, Biomacromolecules, 2012, 13, 1523-1532.

25 G. K. Such, J. F. Quinn, A. Quinn, E. Tjipto and F. Caruso, J. Am. Chem. Soc., 2006, 128, 9318-9319.

26 T. Serizawa, K. Nanameki, K. Yamamoto and M. Akashi, Macromolecules, 2002, 35, 2184-2189.
27 R. J. Russell, K. Sirkar and M. V. Pishko, Langmuir, 2000, 16, 4052-4054.

28 L. Richert, F. Boulmedais, P. Lavalle, J. Mutterer, E. Ferreux, G. Decher, P. Schaaf, J. C. Voegel and C. Picart, Biomacromolecules, 2004, 5, 284-294.

29 J. Groll, J. Fiedler, E. Engelhard, T. Ameringer, S. Tugulu, H. A. Klok, R. E. Brenner and M. Moeller, J. Biomed. Mater. Res., Part A, 2005, 74A, 607-617.

30 J. Groll, T. Ameringer, J. P. Spatz and M. Moeller, Langmuir, 2005, 21, 1991-1999.

31 H. Götz, U. Beginn, C. F. Bartelink, H. J. M. Grünbauer and M. Möller, Macromol. Mater. Eng., 2002, 287, 223-230.

32 P. D. Dalton, C. Hostert, K. Albrecht, M. Moeller and J. Groll, Macromol. Biosci., 2008, 8, 923-931.

33 S. Sinn, M. Eichler, L. Mueller, D. Buenger, J. Groll, G. Ziemer, F. Rupp, H. Northoff, J. Geis-Gerstorfer, F. K. Gehring and H. P. Wendel, Sensors, 2011, 11, 52535269.

34 M. V. Beer, C. Rech, S. Diederichs, K. Hahn, K. Bruellhoff, M. Moller, L. Elling and J. Groll, Anal. Bioanal. Chem., 2012, 403, 517-526. 